\title{
22 Selbstschutz und Selbstgefährdung als ethischer Konflikt in der Notfallsituation
}

\author{
Hartwig Marung
}

\subsection{Einleitung}

Die Arbeitsbedingungen in der Notfallmedizin, und hier vor allem im Rahmen der präklinischen Versorgung, sind u.a. gekennzeichnet durch hohen Handlungsdruck, Informationsmangel bezüglich anamnestischer Daten, widrige äußere Bedingungen wie Kälte/Regen oder Hitze, Stressfaktoren wie eine Vielzahl von Folgeeinsätzen oder umgekehrt sehr lange einsatzfreie Zeiten, ungünstige Tageszeit etc. (vgl. Kap. 2).

Hinzu kommen regelmäßig Notfallsituationen in Grenzbereichen, aus denen eine Reihe weiterer potenzieller Gefahren für die Einsatzkräfte resultiert. Auch hier ist in erster Linie die außerklinische Notfallmedizin zu nennen, weil die meisten Gefährdungen bis zur Aufnahme des oder der Patienten in der Notfallaufnahme bereits identifiziert und bewältigt bzw. verringert werden können. Eine Ausnahme bilden bestimmte gravierende Infektionskrankheiten und, in Deutschland bisher selten, die Verlagerung bewaffneter Auseinandersetzungen vom Einsatzort bis in die Räumlichkeiten der Notaufnahme, wie sie in den USA seit mehreren Jahrzehnten immer wieder vorkommen. Um eine entsprechende Selbstgefährdung des Personals zu reduzieren, ist die Vorhaltung von bewaffnetem Sicherheitspersonal in den Notaufnahmen amerikanischer Großstädte mittlerweile ebenso selbstverständlich wie das Tragen schuss- und stichsicherer Westen für das Rettungsfachpersonal; ein Schritt, der hierzulande (noch) eine Rarität darstellt.

Fälle, in denen von einer Selbstgefährdung der Rettungskräfte auszugehen ist, bilden glücklicherweise die Minderheit aller Notfalleinsätze (Schlechtriemen et al. 2014). Dennoch darf bei diesem Thema nicht ausschließlich an seltene Einsatzanlässe wie Geiselnahmen, Brandeinsätze oder Gewaltverbrechen gedacht werden. Auch Einsätze bei Patienten mit unklaren bzw. schwer zu behandelnden Infektionserkrankungen 
können eine Gefahr für die Gesundheit der Rettungskräfte darstellen, ebenso wie die alltäglich tausendfach durchgeführten Fahrten unter Nutzung von Sonder- und Wegerechten zum Einsatzort oder in eine geeignete Klinik. Für alle diese Aspekte müssen innerbetriebliche und in das Gesamtsystem passende Sicherheitsstandards entwickelt werden. Die Verantwortung hierfür liegt bei den obersten Leitungen, z.B. den Verantwortlichen auf Trägerseite, der Geschäftsführung von Hilfsorganisationen und Kliniken, sowie der ärztlichen Leitung der Notarztstandorte. Die entsprechenden Prozesse sollten Teil der Bemühungen zum Aufbau einer aktiven Sicherheitskultur in der Notfallmedizin sein.

Werden die genannten Risiken für Einsatzkräfte nicht ausreichend bewältigt, können daraus fehlerhafte Patientenversorgungen (vgl. Kap. 24), eigene Gesundheitsstörungen, erhöhte Fehlzeiten und Suchtverhalten bis hin zum Ausscheiden aus dem Beruf resultieren. Die Beschäftigung damit sollte daher nicht nur im Hinblick auf die Patientensicherheit, sondern auch auf Mitarbeitersicherheit und Personalentwicklung stattfinden (Bengel $u$. Heinrichs 2004).

\section{Merke:}

Aus diesen einleitenden Sätzen folgt, dass die Vorgesetzten und Mitarbeitenden aller Berufsgruppen innerhalb der Notfallmedizin das Thema „Selbstgefährdung im Einsatz" nicht ignorieren sollten.

\subsection{Normative Vorgaben}

\subsubsection{Gesetzliche Regelungen}

Rechtsnormen im Hinblick auf Selbstschutz bzw. -gefährdung im Notfalleinsatz liegen in Form von Unfallverhütungs-Vorschriften und der Arbeitsschutz-Gesetzgebung vor. Daneben sind für alle Beteiligten die Beschränkungen der wöchentlichen Arbeitsstunden durch das Arbeitszeitgeset ${ }^{11}$ relevant, die nur in Ausnahmefällen überschritten werden dürfen.

Die Regelungen der StVO ${ }^{12}$ für die Durchführung von Einsatzfahrten und die Richtlinie des Robert-Koch-Instituts zur Hygiene im Krankentransport (Robert Koch-Institut 2007) enthalten ebenfalls verbindliche Vorgaben für den Schutz von Patienten, Mitarbeitern und Dritten und sind daher unbedingt zu beachten.

Umgekehrt wird jeder Mitarbeiter im Rettungsdienst, Notaufnahme und Notarztdienst regelmäßig über die Themen „Garantenstellung“ und „unterlassene Hilfeleistung“ belehrt (vgl. Kap. 7). Aus dieser Konstellation können Konflikte zwischen Selbstschutz und Verpflichtung zur Hilfe resultieren.

Die Pflicht zur Hilfeleistung nach $\mathbb{3} 323 \mathrm{C}$ StGB ist dann gegeben, wenn die Hilfe in einer Notfallsituation sowohl erforderlich als auch zumutbar ist, wobei eine „erhebliche eigene Gefahr“ diese Verpflichtung abmildert ${ }^{13}$. Nachfolgend aufgeführtes Einsatzbeispiel soll diese Aspekte verdeutlichen:

11 http://www.gesetze-im-internet.de/bundesrecht/arbzg/gesamt.pdf (Zugriff am 01.06.2015)

$12 \mathrm{http}: / /$ www.gesetze-im-internet.de/bundesrecht/stvo_2013/gesamt.pdf (Zugriff am 01.06.2015)

13 http://www.gesetze-im-internet.de/bundesrecht/stgb/gesamt.pdf (Zugriff am 01.06.2015) 


\begin{abstract}
Einsatzbeispiel
Mehrere Fahrzeuge des Rettungsdienstes werden zu einem Einsatz in einem städtischen Gymnasium disponiert. Dort soll eine noch unbekannte Anzahl von Verdächtigen Schusswaffen eingesetzt haben; die Zahl der verletzten Schüler und Lehrkräfte ist zum Zeitpunkt der Alarmierung nicht bekannt. Polizeikräfte sind offenbar noch nicht vor Ort. Die Leitstelle weist die Fahrzeuge zur Aufstellung in einem Bereitstellungsraum in 200 Metern Entfernung vom Einsatzort an; ein Vorrücken zum eigentlichen Notfallort soll zunächst nicht erfolgen.
\end{abstract}

Die Einsatzmeldung lässt auf ein besonders zeitkritisches Notfallereignis schließen: Betroffene könnten potenziell lebensbedrohliche Verletzungen wie von außen nicht stillbare Blutungen innerer Organe erlitten haben, die einer sofortigen operativen Behandlung in einer Klinik bedürfen. Die gleichzeitig bestehenden Gefahren für die Rettungskräfte lassen dagegen zunächst nur ein abwartendes Handeln zu, und an diesem kritischen Punkt kann ein ethischer Konflikt gegeben sein. Abgemildert wird dieser durch die klare Weisung der Leitstelle, zunächst nicht den Einsatzort anzufahren; zudem ist, wie im Gesetz aufgeführt, von einer erheblichen eigenen Gefährdung auszugehen, sodass der Vorwurf der unterlassenen Hilfeleistung nicht greifen würde. Grundsätzlich lassen sich derartige Konflikte nur lösen, wenn Sicherheitsstandards definiert wurden und in der spezifischen Situation von allen Beteiligten eingehalten werden. Auf diesen Aspekt wird in Kapitel 22.3 detailliert eingegangen.

\title{
Merke:
}

Kein Mitarbeiter darf sich aus Angst vor dem Vorwurfeiner unterlassenen Hilfeleistung in Gefahr begeben!

\subsubsection{Aus- und Weiterbildungsordnungen}

\section{Regelungen für das Rettungsfachpersonal}

Zu den wichtigsten Voraussetzungen für sachgerechtes Handeln, insbesondere unter hohem Zeitdruck, gehören die qualifizierte Erstausbildung bzw. Weiterbildung und regelmäßige, strukturierte Fortbildungs-Veranstaltungen.

Im Rahmen der Erstausbildung von Rettungsfachpersonal wurde die in diesem Kapitel beschriebene Thematik jahrzehntelang kaum berücksichtigt. Ausnahmen bildeten die vor allem in den nördlichen Bundesländern mit der Notfallrettung beauftragten Berufsfeuerwehren, die ihren Mitarbeitern die Grundsätze für den Umgang mit Gefahren am Einsatzort im Rahmen der feuerwehrtechnischen Ausbildung vermittelten.

In der neuen Ausbildungs- und Prüfungsverordnung (APrV) ${ }^{14}$, die im Hinblick auf das Berufsbild des Notfallsanitäters erarbeitet wurde, sind die Begriffe „Selbstschutz“, „Selbstgefährdung“ und „Ethik“ nicht enthalten. Dennoch finden sich entsprechende Inhalte, wenn z.B. zu dem Themenbereich „Notfallsituationen bei Menschen aller Altersgruppen sowie Gefahrensituationen erkennen, erfassen und bewerten“ aus-

14 http://www.gesetze-im-internet.de/notsan-aprv/index.html (Zugriff am 01.06.2015) 
geführt wird, dass die Schülerinnen und Schüler dazu befähigt werden sollen, „unter Beachtung der Lage vor Ort und möglicher Gefahren Maßnahmen zur Erkundung einer Einsatzstelle durchzuführen“ und „die eigenen Grenzen insbesondere im Hinblick auf die Gefährdungslage, die Zahl der betroffenen Personen oder die berufsrechtlichen Rahmenbedingungen zu beachten." (Hervorhebungen durch den Autor).

Diesbezügliche Hinweise finden sich noch in drei weiteren Themenbereichen innerhalb der APrV, sodass davon auszugehen ist, dass die angehenden Notfallsanitäter im Hinblick auf Selbstschutz und Selbstgefährdung umfassend vorbereitet sein werden.

Ebenso wichtig ist es, für die bereits ausgebildeten Mitarbeitenden im Rettungsdienst passende Fortbildungs-Angebote zu schaffen, mit deren Hilfe eine entsprechende Sensibilisierung erfolgen kann: Die meisten Bundesländer sehen pro Mitarbeiter einen Fortbildungsumfang von 30 Stunden jährlich vor. Die Teilnahme wird als Arbeitszeit angerechnet und die entsprechenden Programme werden von den Kostenträgern refinanziert. Neben Pflicht-Bestandteilen wie Hygiene und Arbeitsschutz können die Aufgabenträger fakultative Anteile, auch zu Themen der Ethik in der Notfallmedizin berücksichtigen.

\section{Regelungen für Notärzte}

Die kürzlich angepassten Inhalte für die Weiterbildung zum Notarzt berücksichtigen ebenfalls entsprechende Inhalte. So widmet sich der Abschnitt 6 des Kursbuchs Notfallmedizin (Bundesärztekammer 2014) der „Erstversorgung unter erschwerten Bedingungen“" (s. Tab. 26).

Im Abschnitt 28 „Psychosoziale Notfälle, Krisenintervention“ werden den angehenden Notärzten die Grundlagen dieser Themenbereiche mit dem Ziel vermittelt, dass diese „die Ursachen und Besonderheiten, auch hinsichtlich ihres eigenen Verhaltens im Einsatz, bei psychosozialen Notfällen im Notarztdienst und ggf. zur sofortigen Krisenintervention erlernen."

\section{Tab. 26 Erschwerende Faktoren bei der Patientenversorgung (zitiert nach Bundesärztekammer 2014)}

\begin{tabular}{|c|c|}
\hline Hindernis & Beispiel \\
\hline $\begin{array}{l}\text { Umgebungsbedingungen } \\
\text { Notfallort }\end{array}$ & $\begin{array}{l}\text { z.B. Wasser, Wald, Sumpf, Steilhang, Kälte, Hitze } \\
\text { Distanz Notfallort - Rettungsmittel } \\
\text { Gefährdende Umgebung (Feuer, gefährliche Stoffe und Güter, Gas, Strom, } \\
\text { Einsturzgefahr, Silo etc.) }\end{array}$ \\
\hline $\begin{array}{l}\text { Erschwerter Zugang zum } \\
\text { Patienten }\end{array}$ & $\begin{array}{l}\text { Einklemmung bei z.B. Verkehrs-, Industrie-, Baustellenunfall, } \\
\text { Inadäquate Behandlungsräumlichkeit (Enge, Dunkelheit, Lärm, Großveran- } \\
\text { staltung, Zusatzgefahren etc.) }\end{array}$ \\
\hline Soziale Faktoren & $\begin{array}{l}\text { Nicht kooperationsfähiger Patient (Hypoglykämie, Intoxikation, Psychose) } \\
\text { Nicht kooperationswilliger Patient (Behandlungs-/Informationsverweige- } \\
\text { rung, Transportverweigerung) } \\
\text { Aggressiver Patient, aggressives Milieu (Kriminalität, Alkohol, Drogen etc.) }\end{array}$ \\
\hline Inadäquate Erwartungen & Patient, Angehörige, Kollegen, Behörden etc. \\
\hline $\begin{array}{l}\text { Inadäquate Ausstattung der } \\
\text { Rettungsmittel, inadäquate } \\
\text { Assistenz }\end{array}$ & \\
\hline
\end{tabular}


Erstmalig wird auch der Punkt „Folgen fehlerhafter Behandlungen für den Mitarbeiter“ in diesem Kursbuch diskutiert (s. Abschnitt „Second Victim“).

Abschließend greift auch der einstündige theoretische Abschnitt „Einsatztaktik“/ Koordination der medizinischen mit der technischen Rettung “ entsprechende Inhalte wie das Arbeiten in Gefährdungsbereichen (z.B. Feuer, Explosionsgefahr, gefährliche Stoffe und Güter, Elektrizität, Strahlung, Ein- oder Absturzgefährdung) auf.

\section{Merke:}

Die Sensibilisierung von Fachpersonal und Notärzten ist elementar, damit alle Mitglieder des Rettungsteams am Einsatzort sich der spezifischen Gefahren bewusst sind.

Aus diesem Grund sollten auch für bereits langjährig im Einsatz befindliche Notärzte entsprechende Fortbildungsinhalte angeboten werden.

\subsection{Einhalten von Sicherheitsstandards}

Allen Sicherheitsstandards ist, wie eingangs dargestellt, gemeinsam, dass diese Vorgaben ihre Wirkung nur entfalten können, wenn sie im Realeinsatz befolgt werden. Die wichtigste Frage lautet daher: Wie kann eine Umsetzung unter den schwierigen Bedingungen der Notfallmedizin erfolgen?

Diese Frage lässt sich am besten beantworten, wenn der Prozess der Notfallrettung in drei Abschnitte unterteilt wird: in die Phase vor dem Einsatz; den eigentlichen Notfalleinsatz und drittens die Phase der Einsatznachbereitung.

\subsubsection{Vermittlung von Sicherheitsstandards im Rahmen der Einsatzvorbereitung}

Die Verantwortung in Bezug auf die Sicherheit am Arbeitsplatz liegt bei der obersten Leitung und ist nicht delegierbar. Das bedeutet nicht, dass diese sämtliche Prozesse selbst erarbeiten und vermitteln muss. Sie muss jedoch die Bedingungen dafür schaffen, dass dieses passiert.

Neben juristischen Normen bilden viele standardisierte Kurskonzepte, zum Beispiel zur Versorgung Schwerverletzter, Sicherheitsstandards ab; häufig an exponierter Stelle am Beginn des entsprechenden Versorgungs-Algorithmus. Diese Standards sind auch in die im Zuge der Einführung des neuen Berufsbildes „Notfallsanitäter“ erstellten Algorithmen für die präklinische Versorgung integriert worden. Beispielhaft sei hier die landesweit gültigen Vorgaben der „Arbeitsgemeinschaft Notfallsanitäter-Umsetzung Niedersachsen; AG NUN“ (2014) genannt (s. Abb. 11). Ähnliche Inhalte finden sich in den weit verbreiteten Therapieempfehlungen der Arbeitsgemeinschaft in Norddeutschland tätiger Notärzte (AGNN 2015) und den Regelungen vieler anderer Bundesländer. Aufgabe der Rettungsdienst-Träger, vertreten durch den Ärztlichen Leiter Rettungsdienst (ÄLRD) und der Schulen ist es, diese Algorithmen, ggf. angepasst an regionale Besonderheiten gemeinsam mit den Beauftragten im Rettungsdienst zu implementieren und deren Einhaltung mittels Maßnahmen des Qualitätsmanagements zu überprüfen. 


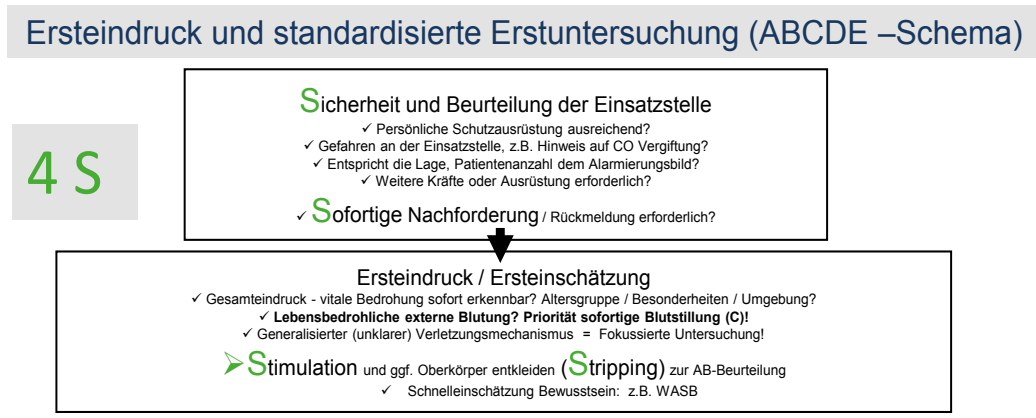

Abb. 11 Auszug aus den Rahmen-Algorithmen der Ag NUN (2014, mit freundlicher Genehmigung)

\subsubsection{Einhaltung von Sicherheitsstandards im Einsatz}

Damit Einsatzkräfte sich trotz hohen Handlungsdrucks, z.B. nach einem Gewaltverbrechen, nicht in Gefahr begeben, sollten einige Prinzipien beachtet werden, deren Anwendung grundsätzlich in jedem Notfalleinsatz sinnvoll und möglich ist. Der Oberbegriff, unter dem diese Prinzipien zusammengefasst werden, lautet „Crew Resource Management“ (CRM); von einigen Autoren wird auch die alternative Begrifflichkeit des „Crisis ...“ oder „Team Resource Management“ favorisiert.

Die sog. „CRM-Pyramide“ (s. Abb. 12) stellt die Einzel-Komponenten dieses Konzepts dar (Rall et al. 2013).

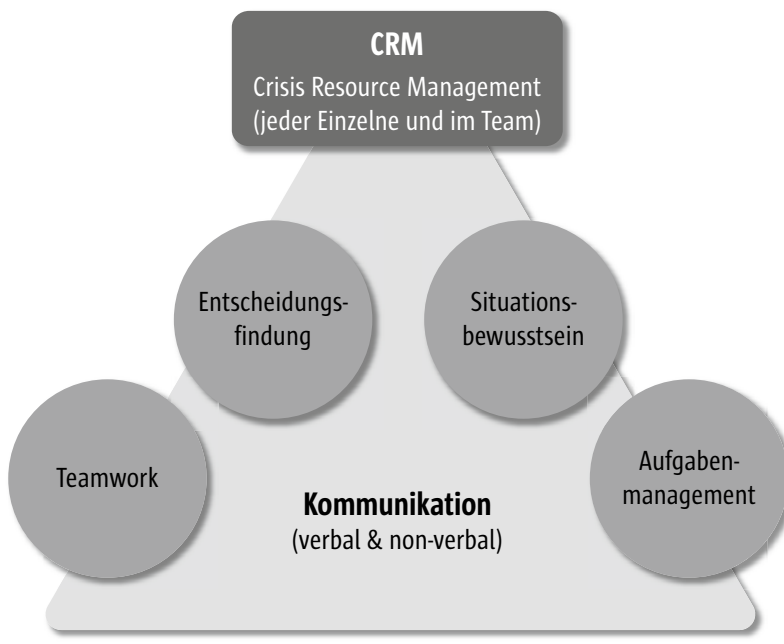

\section{Sicherheitskultur}

Patientensicherheit als höchste Priorität, Systemsicherheit, organisationales Lernen, Elemente der High Reliability Organisationen (HRO), hohe Priorität für optimale Teamarbeit

Abb. 12 Die CRM-Pyramide (nach Rall u. Lackner 2010, mit freundlicher Genehmigung der SpringerVerlag $\mathrm{GmbH}$ ) 
Eines der Kernprinzipien des CRM, die sog. „10-für-10-Regel“ (s. Abb. 13), ist im Hinblick auf den Selbstschutz des Einsatzpersonals besonders hilfreich: Diese bezeichnet ein kurzes (zehn Sekunden) gedankliches Zurücktreten aus der Akutsituation mit dem Ziel, die erforderlichen Schritte für die nächsten zehn Minuten zu durchdenken und innerhalb des Teams abzustimmen. Auf diese Weise werden Fixierungsfehler vermieden, aber auch Gefährdungen können kurz reflektiert und das daraus resultierende Vorgehen kann abgestimmt werden.

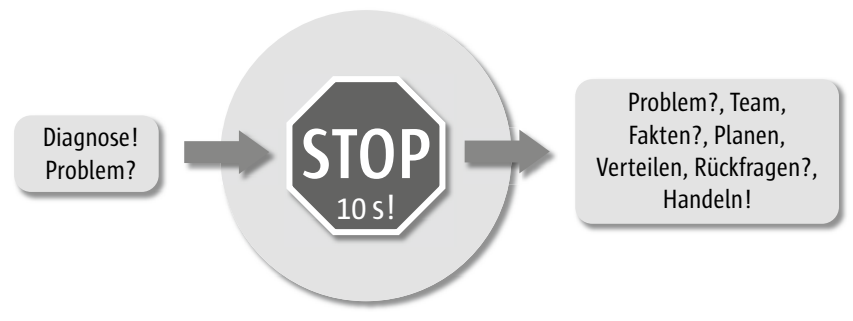

Abb. 13 Zehn-für Zehn-Regel (nach Rall u. Lackner 2010, mit freundlicher Genehmigung der SpringerVerlag $\mathrm{GmbH}$ )

Daneben favorisiert das CRM-Prinzip den Einsatz von Checklisten, der bei richtiger Umsetzung die Sicherheit in der operativen Medizin signifikant erhöht (Haynes et al. 2009) und auch in der Notfallmedizin praktikabel ist (Marung et al. 2014). Zudem propagieren die Autoren das frühzeitige Hinzuziehen von personeller Unterstützung; dieses ist im Notfalleinsatz zwar nicht so einfach umsetzbar wie unter klinischen Bedingungen. Dennoch kann die situationsgerechte Hinzuziehung von Feuerwehr, Polizei, Pädiater, Giftnotruf etc. zur Erhöhung der Sicherheit und damit der eigenen Handlungsfähigkeit beitragen. Eine weitere taktische Maßnahme zur Erhöhung der eigenen Sicherheit ist die Beschränkung der Erstversorgung am Notfallort; insbesondere bei Einsätzen, in denen eine Kontamination der Einsatzstelle oder sogar ein physischer Angriff auf die Helfer nicht ausgeschlossen werden können.

Dass in diesem Zusammenhang auch eine funktionierende Teamarbeit von großer Bedeutung ist, liegt auf der Hand. Im Detail wird dieses Thema in Kapitel 17 dargestellt.

\subsubsection{Sicherheitsstandards und Einsatznachbereitung}

Nachbesprechungen kritischer Einsätze sind in Deutschland noch nicht flächendeckend etabliert, haben sich aber als Instrument zur Aufarbeitung gut bewährt. Sie können entweder direkt im Anschluss an einen Notfalleinsatz erfolgen („,hot debriefing“) oder mit etwas zeitlichem Abstand („cold debriefing“). Beide Methoden haben spezifische Vor- und Nachteile (Couper et al. 2013): Besprechungen unmittelbar nach dem Einsatz finden immer unter dem Vorbehalt der Alarmierung zu einem Folgeeinsatz statt. Zudem kann es den Beteiligten schwer fallen, das gerade Erlebte objektiv $z u$ bewerten. Besprechungen mit zeitlichem Abstand können organisatorisch schwierig zu organisieren sein, z.B. aufgrund von unterschiedlichen Schichtplänen. 
Merke:

Je komplexer die Einsatzsituation, desto wichtiger ist es, dass eine Besprechung stattfindet und von hierfür ausgebildeten Kräften, z.B. der psychosozialen Notfallversorgung (PSNV) geleitet wird.

Wichtige Hinweise und Empfehlungen hierzu finden sich in einer Publikation des Bundesamts für Bevölkerungsschutz und Katastrophenhilfe (2012).

Die regelmäßige Durchführung von Fallbesprechungen, die in vielen Kliniken mittlerweile realisiert wird, kann auch im Bereich der Notfallmedizin dazu beitragen, dass eine größere Anzahl von Mitarbeitern aus kritischen Einsätzen lernt, auch wenn sie persönlich nicht unmittelbar beteiligt waren (Teixeira et al. 2007). Auch die anonyme Meldung kritischer Ereignisse, z.B. im Rahmen sog. „Critical Incident Reporting Systeme“ (CIRS) führt zu mehr Transparenz in Bezug auf selbstgefährdende Ereignisse (Hohenstein et al. 2014). Insofern ist es zu begrüßen, dass im Jahr 2015 in Bayern ein Modellprojekt zur landesweiten Erfassung kritischer Ereignisse im Rettungsdienst initiiert wurde, das vom Institut für Notfallmedizin und Medizin-Management (INM) der Ludwig-Maximilians-Universität München wissenschaftlich begleitet wird.

\section{4 Überforderung des Retters}

Überforderung entsteht, wenn die Kompensationsmechanismen eines Individuums erschöpft sind. Dass dieses eintritt, kann selbst bei Einhaltung aller oben beschriebenen Maßnahmen niemals völlig ausgeschlossen werden. Die Folgen für den Betroffenen sind u.a. Konzentrationsprobleme, Reizbarkeit und Schlafstörungen, die in einer Einengung des Denkens und Verhaltens, der sog. „kognitiven Notfallreaktion“ münden können (St. Pierre et al. 2011). Hierbei nehmen die Betroffenen in komplexen Situationen unbewusst Vereinfachungen und Fehlbewertungen vor, was $\mathrm{zu}$ falschen diagnostischen und therapeutischen Maßnahmen und damit zu einer Gefährdung von Patienten, Mitarbeitern und Dritten führen kann.

Wichtig ist, dass in derartigen Fällen professionell und im Sinne aller Beteiligten gehandelt wird, wobei auch hier die Verantwortung in erster Linie bei der Leitung der betreffenden Organisation liegt.

Ein Sonderfall liegt dann vor, wenn ein Mitarbeiter infolge eines ihm selbst unterlaufenen Fehlers in eine Überforderungssituation gerät, er also zum „zweiten Opfer“ (engl. „second victim“, Wu 200o) wird. Für derartige Situationen haben erste medizinische Fachgesellschaften Handlungsempfehlungen entwickelt, die in vorbildlicher Weise die erforderlichen Maßnahmen beschreiben (Bock et al. 2013).

Kernbotschaften für die Praxis

- Rettungsfachpersonal und Notärzte sollen gleichermaßen für das Thema "Selbstgefährdung und Selbstschutz" sensibilisiert werden.

- Die Verantwortung hierfür liegt bei der Leitung der Institution, die den Mitarbeiter beschäftigt. 


\section{- Die Anwendung strukturierter Maßnahmen im Rahmen der Einsatzvorbe- reitung, im Einsatz selbst und in der Phase der Einsatznachbereitung kann das Sicherheitsniveau nachhaltig erhöhen.}

\section{Literatur}

Arbeitsgemeinschaft in Norddeutschland tätiger Notärzte (AGNN) (2015) Therapieempfehlungen für die Notfallmedizin. Verlag Schmidt-Römhild, Lübeck

Arbeitsgemeinschaft Niedersächsische Umsetzung Notfallsanitäter (AG NUN) (2014) Aus- und Fortbildung Notfallsanitäter. Anlage Rahmen-Algorithmen Niedersachsen. www.mi.niedersachsen.de/download/92677 (Zugriff am 01.06.2015)

Bengel J, Heinrichs M (2004) Psychische Belastungen des Rettungspersonals. In: Bengel ) (Hrsg.) Psychologie in Notfallmedizin und Rettungsdienst. 2. Auflage. Springer, Berlin, Heidelberg

Bock RW, Biermann E, Wulff H (2013) Umgang mit schweren Behandlungskomplikationen und belastenden Verläufen. Anästh Intensivmed 54: 490-494

Bundesamt für Bevölkerungsschutz und Katastrophenhilfe (2012) Psychosoziale Notfallversorgung. Qualitätsstandards und Leitlinien (Teil I und II). 3. Auflage, Bonn

Bundesärztekammer (2014) Muster-Kursbuch Notfallmedizin. http://www.bundesaerztekammer.de/fileadmin/ user_upload/downloads/MKBNotfallmedizin2014.pdf (Zugriff am 01.06.2015)

Couper K, Salman B, Soar I, Finn I, Perkins GD (2013) Debriefing to improve outcomes from critical illness: a systematic review and meta-analysis. Intensive Care Med; 39: 1513-1523

Haynes AB, Weiser TG, Berry WR, Lipsitz SR, Breizat A-HS, Dellinger EP, Herbosa T, Joseph S, Kibatala PL, Lapitan MCM, Merry AF, Moorthy K, Reznick RK, Taylor B, Gawande AA; for the Safe Surgery Saves Lives Study Group (2009) A surgical safety checklist to reduce morbidity and mortality in a global population. N Engl I Med 29; 360: 491-499

Hohenstein C, Hempel D, Schultheis K, Lotter 0, Fleischmann T (2014) Critical incident reporting in emergency medicine: results of the prehospital reports. Emerg Med J; 31: 415-418

Marung H, Schmidbauer W, Tietz M, Genzwuerker, Kerner T (2014) Use of checklists facilitates guideline adherence in prehospital emergency care. Resuscitation 85: 665

Rall M, Koppenberg I, Hellmann L, Henninger M (2013) Crew Resource Management und Human Factors. In: Moecke H, Marung H, Oppermann S (Hrsg.) Praxishandbuch Qualitäts- und Risikomanagement im Rettungsdienst. Medizinisch-Wissenschaftliche Verlagsgesellschaft, Berlin, S. 149-157

Rall M, Lackner CK (2010) Crisis Resource Management (CRM). Der Faktor Mensch in der Akutmedizin. Notfall Rettungsmed 13: 349-356

Robert Koch-Institut (2007) Anforderungen der Hygiene an den Krankentransport einschließlich Rettungstransport in Krankenkraftwagen. In: Richtlinie für Krankenhaushygiene und Infektionsprävention. Elsevier, Urban \& Fischer, München

Schlechtriemen T, Armbruster W, Adler I, Bartha C, Becker K, Höhn M, Kubulus D, Morbe A, Reeb R, Schmidt D, Steiner P, Wrobel M (2014) Herausforderung Notarztdienst - Weiterbildungskonzept für ein anspruchsvolles ärztliches Tätigkeitsfeld. Notfall \& Rettungsmedizin 17(1): 39-45

St. Pierre M, Hofinger G, Buerschaper C (2011) Stress: Ärzte unter Strom. In: St. Pierre M, Hofinger G, Buerschaper C (Hrsg.) Notfallmanagement - Human Factors und Patientensicherheit in der Akutmedizin. 2. Auflage. Berlin, Heidelberg, Springer, S. 133-152

Teixeira PG, Inaba K, Hadjizacharia P, Brown C, Salim A, Rhee P, Browder T, Noguchi TT, Demetriades D (2007) Preventable or potentially preventable mortality at a mature trauma center. J Trauma 63(6): 1338-46

Wu AW (2000) Medical error: the second victim. The doctor who makes the mistake needs help too. BMI 320: $726-727$ 
22 Selbstschutz und Selbstgefährdung als ethischer Konflikt in der Notfallsituation

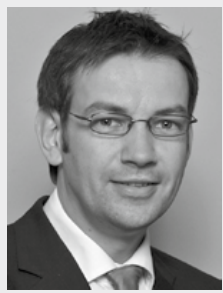

Dr. med. Hartwig Marung

Studium der Humanmedizin in Marburg und Kiel. Ausbildung zum Facharzt für Anästhesiologie am Städtischen Klinikum Braunschweig. Von 2011-2015 Oberarzt am Institut für Notfallmedizin der Asklepios Kliniken Hamburg. Seit September 2015 Oberarzt und Leiter des Bereichs Qualitätsmanagement am Institut für Rettungs- und Notfallmedizin des Universitätsklinikums SchleswigHolstein (UKSH). Schwerpunkte: Qualität und Sicherheit in der Notfallmedizin. 